hep-th/0105007

EFI-2000-53

\title{
Can we live in a self-tuning universe?
}

\author{
Sean M. Carroll ${ }^{1}$ and Laura Mersini ${ }^{2}$ \\ ${ }^{1}$ Enrico Fermi Institute and Department of Physics, University of Chicago \\ 5640 S. Ellis Avenue, Chicago, IL 60637, USA \\ carroll@theory . uchicago.edu \\ ${ }^{2}$ Scuola Normale Superiore, Piazza dei Cavalieri 7, I-56126 Pisa, Italy \\ mersini@cibs.sns.it
}

\begin{abstract}
The self-tuning brane scenario is an attempt to solve the cosmological constant problem in the context of extra dimensions. Rather than making the vacuum energy small, this approach proceeds by removing the gravitational effect of vacuum energy on the expansion of the universe. Such behavior is only possible through changing the Friedmann equation of conventional cosmology, and we discuss difficulties in obtaining cosmological evolution compatible with observation in this context. Specific models considered include a bulk scalar field coupling to the brane via a conformal transformation of the brane metric, and via a rescaling of the brane volume element.
\end{abstract}




\section{Introduction}

The fact that the observed cosmological constant is much smaller than the expected value [1, 2, 2] may provide a crucial clue in our attempts to understand the nature of spacetime. Most attempts to solve the problem can be characterized as making the vacuum energy much smaller than its natural value. Alternatively, however, we can imagine keeping a large vacuum energy, but changing the gravitational dynamics in such a way that the vacuum does not act as a (significant) source of spacetime curvature. Since it is only through its gravitational influence that the vacuum energy can be measured, such an arrangement could reconcile the naive estimates $\rho_{\mathrm{vac}} \geq\left(10^{18} \mathrm{GeV}\right)^{4}$ with the observationally favored result $\rho_{\text {vac }} \sim\left(10^{-3} \mathrm{eV}\right)^{4}$ 때, [3].

The idea of brane-worlds and large extra dimensions [5] opens up a new set of ways to think about the cosmological constant problem. In these scenarios, our observed fourdimensional theory of gravity is descended from a higher-dimensional embedding, and in principle the resulting dynamics can differ dramatically from a straightforward four-dimensional expectation.

An example of such an altered dynamics is provided by the idea of self-tuning branes [6, 7]. Here, matter fields on a three-brane with a single extra dimension are coupled to a bulk scalar field. With an appropriate choice of couplings, Minkowskian solutions on the brane can be found with any brane cosmological constant. In this paper, we put aside fundamental issues of the feasibility of the self-tuning idea (e.g., the role of singularities) to concentrate on whether this kind of scenario can be made compatible with conventional cosmology. (See [8, 9] for investigations of brane-world cosmology, and [10, 11] for studies of the self-tuning scenario.)

On a self-tuning brane, there is vacuum energy (essentially the tension of the brane), but the spacetime geometry is nevertheless flat. Somehow, then, the geometry is insensitive to the vacuum energy. It is necessary, however, that spacetime respond to at least some sorts of energy density; in particular, the success of Big Bang Nucleosynthesis (BBN) [12] provides evidence in favor of the standard picture in the radiation-dominated era. It is therefore necessary to recover at least some portion of conventional cosmology, while removing the effects of the cosmological constant.

In this work we attempt to characterize the empirical challenges to a successful self-tuning cosmology. In the next section we discuss in general how the self-tuning mechanism may be understood in terms of the energy and pressure on the brane, pointing out the distinctions 
with conventional Friedmann cosmology. We then consider two specific models of self-tuning, and derive effective Friedmann-like equations relating the Hubble parameter to the energy and pressure. In section 4 we compare these models with what we know about the universe, and discuss whether they may be brought into agreement with observation. Although such agreement seems unlikely, we are unable to rule it out entirely.

\section{The secret of self-tuning cosmology}

In this section we consider how a theory of gravity may in principle be insensitive to vacuum energy while allowing other forms of energy-momentum to influence spacetime curvature. We consider a flat Robertson-Walker metric in $3+1$ dimensions,

$$
d s^{2}=-d t^{2}+a^{2}(t) d \vec{x}^{2}
$$

where $a(t)$ is the scale factor and $d \vec{x}^{2}$ is the flat Euclidean metric. An energy-momentum tensor consistent with a Robertson-Walker metric will be spatially isotropic, taking the form

$$
T_{\nu}^{\mu}=\operatorname{diag}(-\rho, p, p, p)
$$

where $\rho$ is the energy density and $p$ the pressure. The conventional Friedmann equation of general relativity is then

$$
H^{2} \equiv\left(\frac{\dot{a}}{a}\right)^{2}=\frac{8 \pi G}{3} \rho
$$

where $H$ is the Hubble parameter and $G$ is the (four-dimensional) Newton's constant.

In a self-tuning model, it is possible to find a Minkowski-spacetime solution $(H=0)$ regardless of the value of $\rho_{\mathrm{vac}}$ (when all other energy densities vanish). Clearly, for this to be possible (3) will have to be modified, either in its explicit form or in the definition of $\rho$. If, however, we consider theories which arise from varying a specified action with respect to the metric tensor to derive gravitational field equations, the energy density and pressure (in this coordinate system) are defined by

$$
\rho=-T_{0}^{0}=2 \frac{1}{\sqrt{g}} g^{00} \frac{\partial \mathcal{L}_{\text {matter }}}{\partial g^{00}}, \quad p=T_{1}^{1}=-2 \frac{1}{\sqrt{g}} g^{11} \frac{\partial \mathcal{L}_{\text {matter }}}{\partial g^{11}},
$$

where $g$ is the absolute value of the determinant of the metric and $\mathcal{L}_{\text {matter }}$ is the matter Lagrange density. These definitions do not discriminate between different forms of energy density; there is no way for the gravitational field to tell the difference between energy density from the vacuum and energy density from any other source. 
How, then, can we modify the theory to allow flat solutions in the presence of vacuum energy, while remaining sensitive to the influence of other sources on the expansion rate? Given the metric (11) and energy-momentum tensor (2), we seek an equation written in terms of $\rho, p, a$ and its derivatives (which appear in the curvature tensor), and possibly explicit additional fields. Although vacuum energy should enter any such equation in the same way as other energy, the vacuum does have a distinguishing characteristic, namely its equation of state:

$$
p_{\mathrm{vac}}=-\rho_{\mathrm{vac}} .
$$

With the ingredients at our disposal, this relation suggests a form for a modified Friedmann equation:

$$
H^{2}=f(\rho, p)(\rho+p)+\text { other terms }
$$

where $f(\rho, p)$ is a well-behaved function at $p=-\rho$. Such a relation would allow for a Minkowski solution $(H=0)$ in the presence of arbitrary vacuum energy.

Although (6) differs from the conventional Friedmann law, it is not necessarily incompatible with observation. Consider for example a hypothetical relation

$$
H^{2}=2 \pi G(\rho+p)
$$

During a matter-dominated era, $p=0$, and the Hubble parameter will differ by a factor $\sqrt{3} / 2 \approx 0.87$ for a given value of the energy density. However, since the scaling of $H$ with $\rho$ is as in the conventional theory, and $\sqrt{3} / 2$ is close to unity, it would be hard to distinguish between the behavior predicted by (17) and the usual Friedmann equation during matter domination. During radiation domination, $p=\rho / 3$, and (7) precisely recovers the conventional expectation (by construction). This is important, as our most precise quantitative evidence in favor of conventional cosmology comes from BBN, which occurs while the universe is radiation-dominated.1 Finally, this relation would solve the cosmological constant problem, as $H=0$ when $p=-\rho$.

Unfortunately, we do not have a theory that predicts (7). As we shall see below, the specific self-tuning scenarios we consider lead to additional derivatives of the metric, as well as explicit dependence on the bulk scalar field, and extra terms which are quadratic in the

\footnotetext{
${ }^{1}$ The anisotropy spectrum in the cosmic microwave background (CMB), although it does provide precision constraints on the expansion of the universe, does not do so in a model-independent way. Since a novel theory of gravity which predicted a different Friedmann equation could also predict different behavior for the evolution of large-scale density perturbations, it is impossible to compare directly a phenomenological relationship such as (7) to CMB observations. In any specific full theory, CMB anisotropies are likely to provide an interesting test.
} 
energy and pressure. Generally, it seems unlikely that a theory which did predict a linear dependence of $H^{2}$ on $(\rho+p)$ would both get the correct coefficient $2 \pi G$ (or very close to it) and successfully recover Newton's law $F=G m_{1} m_{2} / r^{2}$ in the solar system. However, this unlikelihood does not seem so great that searching for such a theory would be a waste of time.

\section{Specific Examples}

\subsection{Setup}

We start with an action of the form [6, 7]

$$
S=S_{5}\left[\phi, g^{(5)}\right]+S_{4}\left[\phi, g^{(4)}, \psi_{i}\right]
$$

Here, $S_{5}$ is the bulk action and $S_{4}$ that of the brane; $\phi$ is a scalar field in the bulk with interactions on the brane, while the $\psi_{i}$ 's are matter fields confined to the brane. The metric in the bulk is $g_{a b}^{(5)}$, and we choose coordinates such that the induced metric on the brane is

$$
g_{\mu \nu}^{(4)}=\delta_{\mu}^{a} \delta_{\nu}^{b} g_{a b} .
$$

The indices $a, b$ run over $\{0,1,2,3, y\}$ and $\mu, \nu$ run over $\{0,1,2,3\}$.

The bulk action can be written

$$
S_{5}=\int d^{5} x \mathcal{L}_{5}=\int d^{5} x \sqrt{g^{(5)}}\left(\frac{M_{5}^{3}}{2} R-\alpha(\nabla \phi)^{2}\right),
$$

with $\alpha$ a coefficient which we leave unspecified for the moment, $M_{5}$ is the five-dimensional Planck mass, $R$ is the five-dimensional Ricci scalar, and the brane action as

$$
S_{4}=\int d^{5} x \mathcal{L}_{4}=\int d^{5} x \sqrt{g^{(4)}} \widehat{\mathcal{L}_{4}} \delta(y)=\int d^{4} x \sqrt{g^{(4)}} \widehat{\mathcal{L}_{4}} .
$$

We will leave the actual form of $\widehat{\mathcal{L}_{4}}\left(\phi, g^{(4)}, \psi_{i}\right)$ unspecified in this section, and consider different possibilities in sections 3.2 and 3.3 . We will always consider geometries that have a $\mathbf{Z}_{2}$ symmetry with the brane at the fixed point, so that we need not include a boundary term in the brane action.

The 5-dimensional Einstein's equations are

$$
G_{a b}=M_{5}^{-3} T_{a b}
$$


where the energy-momentum tensor is defined by

$$
T_{a b}=-2 \frac{1}{\sqrt{g^{(5)}}} \frac{\partial \mathcal{L}}{\partial g_{(5)}^{a b}} .
$$

We choose the metric

$$
d s_{(5)}^{2}=-n^{2}(y, t) d t^{2}+a^{2}(y, t) d \vec{x}^{2}+b^{2}(y, t) d y^{2},
$$

where $\vec{x}=\left\{x^{1}, x^{2}, x^{3}\right\}$ are the spatial coordinates along the brane, and $y$ is the transverse spatial dimension. The Einstein tensor is then

$$
\begin{aligned}
G_{00}= & 3\left[\frac{\dot{a}^{2}}{a^{2}}+\frac{\dot{a} \dot{b}}{a b}-\frac{n^{2}}{b^{2}}\left(\frac{a^{\prime \prime}}{a}+\frac{a^{\prime 2}}{a^{2}}-\frac{a^{\prime} b^{\prime}}{a b}\right)\right], \\
G_{0 i}= & 0 \\
G_{0 y}= & 3\left(-\frac{\dot{a}^{\prime}}{a}+\frac{a^{\prime} \dot{b}}{a b}+\frac{\dot{a} n^{\prime}}{a n}\right), \\
G_{i j}= & \frac{a^{2}}{n^{2}}\left(-2 \frac{\ddot{a}}{a}-\frac{\ddot{b}}{b}-\frac{\dot{a}^{2}}{a^{2}}-2 \frac{\dot{a} \dot{b}}{a b}+2 \frac{\dot{a} \dot{n}}{a n}+\frac{\dot{b} \dot{n}}{b n}\right) \delta_{i j} \\
& +\frac{a^{2}}{b^{2}}\left(2 \frac{a^{\prime \prime}}{a}+\frac{n^{\prime \prime}}{n}+\frac{a^{\prime 2}}{a^{2}}-2 \frac{a^{\prime} b^{\prime}}{a b}+2 \frac{a^{\prime} n^{\prime}}{a n}-\frac{b^{\prime} n^{\prime}}{b n}\right) \delta_{i j}, \\
G_{i y}= & 0 \\
G_{y y}= & 3\left[\frac{b^{2}}{n^{2}}\left(-\frac{\ddot{a}}{a}-\frac{\dot{a}^{2}}{a^{2}}+\frac{\dot{a} \dot{n}}{a n}\right)+\frac{a^{\prime 2}}{a^{2}}+\frac{a^{\prime} n^{\prime}}{a n}\right] .
\end{aligned}
$$

The energy-momentum tensor decomposes into contributions from the bulk and the brane,

$$
T_{a b}=T_{a b}^{(5)}+T_{a b}^{(4)}=-2 \frac{1}{\sqrt{g^{(5)}}}\left(\frac{\partial \mathcal{L}_{5}}{\partial g_{(5)}^{a b}}+\frac{\partial \mathcal{L}_{4}}{\partial g_{(4)}^{\mu \nu}} \delta_{a}^{\mu} \delta_{b}^{\nu}\right) .
$$

The components of the bulk $T_{a b}^{(5)}$ receive contributions only from the scalar field $\phi$, given by

$$
\begin{aligned}
T_{00}^{(5)} & =\alpha n^{2}\left(\frac{\dot{\phi}^{2}}{n^{2}}+\frac{\phi^{\prime 2}}{b^{2}}\right) \\
T_{0 y}^{(5)} & =2 \alpha \dot{\phi} \phi^{\prime} \\
T_{i j}^{(5)} & =\alpha a^{2}\left(\frac{\dot{\phi}^{2}}{n^{2}}-\frac{\phi^{2}}{b^{2}}\right) \delta_{i j} \\
T_{y y}^{(5)} & =\alpha b^{2}\left(\frac{\dot{\phi}^{2}}{n^{2}}+\frac{\phi^{\prime 2}}{b^{2}}\right) .
\end{aligned}
$$


The contribution to the energy-momentum tensor from the brane will depend on the chosen brane action; in terms of $\widehat{\mathcal{L}_{4}}$ defined in (11), we have

$$
T_{a b}^{(4)}=\sqrt{\frac{g^{(4)}}{g^{(5)}}}\left(g_{\mu \nu}^{(4)} \widehat{\mathcal{L}}_{4}-2 \frac{\partial \widehat{\mathcal{L}_{4}}}{\partial g_{(4)}^{\mu \nu}}\right) \delta(y) \delta_{a}^{\mu} \delta_{b}^{\nu} .
$$

In addition to Einstein's equations, we need the equation of motion for $\phi$. With the action (10)-(11), the $\phi$ equation is

$$
\nabla_{a} \frac{\partial \mathcal{L}_{5}}{\partial\left(\nabla_{a} \phi\right)}=\frac{\partial \mathcal{L}_{4}}{\partial \phi}
$$

since we will be considering brane actions that depend on $\phi$ but not its derivatives. The left-hand side is

$$
\nabla_{a} \frac{\partial \mathcal{L}_{5}}{\partial\left(\nabla_{a} \phi\right)}=-2 \alpha \sqrt{g_{5}} \square \phi
$$

yielding an equation of motion

$$
\square \phi=-\frac{1}{2 \alpha} \frac{1}{b} \frac{\partial \widehat{\mathcal{L}}_{4}}{\partial \phi} \delta(y),
$$

where $\widehat{\mathcal{L}}_{4}$ is defined by $(11)$ and the D'Alembertian on scalars is given by

$$
\square \phi=-\frac{1}{n^{2}}\left[\ddot{\phi}+\left(-\frac{\dot{n}}{n}+3 \frac{\dot{a}}{a}+\frac{\dot{b}}{b}\right) \dot{\phi}\right]+\frac{1}{b^{2}}\left[\phi^{\prime \prime}+\left(\frac{n^{\prime}}{n}+3 \frac{a^{\prime}}{a}-\frac{b^{\prime}}{b}\right) \phi^{\prime}\right] .
$$

The equations of motion generally involve distributional sources localized on the brane. It is therefore convenient to separate the equations into distinct relations valid in the bulk and on the brane. The bulk equations are simply the full equations with distributional sources omitted; they include the bulk Einstein equations:

$$
\begin{aligned}
3\left[\frac{\dot{a}^{2}}{a^{2}}+\frac{\dot{a} \dot{b}}{a b}-\frac{n^{2}}{b^{2}}\left(\frac{a^{\prime \prime}}{a}+\frac{a^{\prime 2}}{a^{2}}-\frac{a^{\prime} b^{\prime}}{a b}\right)\right] & =\frac{\alpha}{M_{5}^{3}} n^{2}\left(\frac{\dot{\phi}^{2}}{n^{2}}+\frac{\phi^{2}}{b^{2}}\right) \\
3\left(-\frac{\dot{a}^{\prime}}{a}+\frac{a^{\prime} \dot{b}}{a b}+\frac{\dot{a} n^{\prime}}{a n}\right) & =\frac{2 \alpha}{M_{5}^{3}} \dot{\phi} \phi^{\prime} \\
\frac{a^{2}}{n^{2}}\left(-2 \frac{\ddot{a}}{a}-\frac{\ddot{b}}{b}-\frac{\dot{a}^{2}}{a^{2}}-2 \frac{\dot{a} \dot{b}}{a b}+2 \frac{\dot{a} \dot{n}}{a n}+\frac{\dot{b} \dot{n}}{b n}\right) & =\frac{\alpha}{M_{5}^{3}} a^{2}\left(\frac{\dot{\phi}^{2}}{n^{2}}-\frac{\phi^{\prime 2}}{b^{2}}\right) \\
+\frac{a^{2}}{b^{2}}\left(2 \frac{a^{\prime \prime}}{a}+\frac{n^{\prime \prime}}{n}+\frac{a^{\prime 2}}{a^{2}}-2 \frac{a^{\prime} b^{\prime}}{a b}+2 \frac{a^{\prime} n^{\prime}}{a n}-\frac{b^{\prime} n^{\prime}}{b n}\right) & =\frac{\alpha}{M_{5}^{3}} b^{2}\left(\frac{\phi^{2}}{n^{2}}+\frac{\phi^{\prime 2}}{b^{2}}\right),
\end{aligned}
$$


and the bulk scalar field equation

$$
-\frac{1}{n^{2}}\left[\ddot{\phi}+\left(-\frac{\dot{n}}{n}+3 \frac{\dot{a}}{a}+\frac{\dot{b}}{b}\right) \dot{\phi}\right]+\frac{1}{b^{2}}\left[\phi^{\prime \prime}+\left(\frac{n^{\prime}}{n}+3 \frac{a^{\prime}}{a}-\frac{b^{\prime}}{b}\right) \phi^{\prime}\right]=0 .
$$

The corresponding equations on the brane can be expressed without explicit $\delta$-functions by integrating them in the vicinity of the brane to obtain jump conditions [8]. Assuming $\mathbf{Z}_{2}$ symmetry, the jump conditions relate second derivatives with respect to $y$ to the coefficients of $\delta$-function sources. That is, expressions of the form

$$
f^{\prime \prime}(y)=A \delta(y)+B
$$

imply, on the brane,

$$
f_{0}^{\prime}=\frac{1}{2} A
$$

where a subscript 0 indicates that a quantity is evaluated on the brane by taking the limit as $y \rightarrow 0^{+}$. For the scalar field, (29) and (30) imply

$$
\phi_{0}^{\prime}=-\frac{1}{4 \alpha} b_{0}\left(\frac{\partial \widehat{\mathcal{L}}_{4}}{\partial \phi}\right)_{0}
$$

For the metric, it is convenient to express the jump conditions for $a_{0}^{\prime}$ and $n_{0}^{\prime}$ separately for each model, which we do below.

To get a Friedmann-like equation on the brane, we consider the yy component of Einstein's equations (34), evaluated on the brane. (There is no $\delta$-function in $T_{y y}$, so the bulk equation (34) holds true on the brane as well.) We can scale our time coordinate such that $n_{0}=1, \dot{n}_{0}=0$ (although $n$ will generally vary off the brane). We then have

$$
\frac{\ddot{a}_{0}}{a_{0}}+\frac{\dot{a}_{0}^{2}}{a_{0}^{2}}=\frac{a_{0}^{\prime 2}}{a_{0}^{2} b_{0}^{2}}+\frac{a_{0}^{\prime} n_{0}^{\prime}}{a_{0} b_{0}^{2}}-\frac{\alpha}{3 M_{5}^{3}} \frac{\phi_{0}^{\prime 2}}{b_{0}^{2}}-\frac{\alpha}{3 M_{5}^{3}} \dot{\phi}_{0}^{2} .
$$

The next step is to express the spatial derivatives on the right hand side of (39) in terms of energy and momentum on the brane, which requires a specification of the brane Lagrangian $\mathcal{L}_{4}$. In the original papers on self-tuning branes [6, 7], the matter Lagrangian was taken to be a cosmological constant $\lambda$ times an exponential of $\phi$ :

$$
\mathcal{L}_{4}=-\sqrt{g^{(4)}} e^{2 \beta \phi} \lambda \delta(y)
$$

with $\beta$ a coupling constant. In what follows we consider two distinct generalizations of this form to actions with dynamical fields: conformal coupling, in which matter fields on the brane 
couple exclusively to a rescaled metric $\widetilde{g}_{\mu \nu}=e^{\beta \phi} g_{\mu \nu}^{(4)}$, or volume element rescaling, in which the four-dimensional volume element is taken to be $d^{4} x \sqrt{g^{(4)}} e^{2 \beta \phi}$ (and the four-dimensional metric is otherwise simply the induced metric from five dimensions). Both approaches reduce to (40) when brane tension is the only contribution to the four-dimensional action, but give different results for other forms of matter.

\subsection{Conformal coupling}

In this section we consider an action of the form

$$
S=S_{5}\left[\phi, g_{a b}^{(5)}\right]+S_{4}\left[\psi_{i}, e^{\beta \phi} g_{\mu \nu}^{(4)}\right]
$$

where the five-dimensional action is as in (10), and the four-dimensional action is

$$
S_{4}=\int d^{5} x \mathcal{L}_{4}=\int d^{5} x \sqrt{\widetilde{g}} f\left(\psi_{i}, \widetilde{g}_{\mu \nu}\right) \delta(y)=\int d^{4} x \sqrt{\widetilde{g}} f\left(\psi_{i}, \widetilde{g}_{\mu \nu}\right) .
$$

Here, $\psi_{i}$ represents an unspecified set of matter fields, and the metric to which matter on the brane couples is related to the induced metric by a conformal transformation,

$$
\widetilde{g}_{\mu \nu}=e^{\beta \phi} g_{\mu \nu}^{(4)}
$$

equivalent to $\widehat{\mathcal{L}}_{4}=e^{2 \beta \phi} f\left(\psi_{i}, \widetilde{g}_{\mu \nu}\right)$ in the notation defined by (11).

In this model, test particles on the brane move along geodesics of $\widetilde{g}_{\mu \nu}$; this is the only metric perceived by observers on the brane. It therefore is sensible to define all brane quantities in terms of this metric. The energy-momentum tensor as measured by observers living on the brane takes the form

$$
\widetilde{T}_{\mu \nu} \delta(y)=-2 \frac{1}{\sqrt{g}} \frac{\partial \mathcal{L}_{4}}{\partial \widetilde{g}^{\mu \nu}}
$$

The energy density and pressure as seen by brane observers will be, in our coordinate system,

$$
\widetilde{\rho}=-\widetilde{g}^{00} \widetilde{T}_{00}
$$

and

$$
\widetilde{p}=\widetilde{g}^{11} \widetilde{T}_{11}
$$

so we have

$$
\begin{aligned}
\widetilde{T}_{00} & =n^{2} e^{\beta \phi} \widetilde{\rho} \\
\widetilde{T}_{i j} & =a^{2} e^{\beta \phi} \widetilde{p} \delta_{i j} .
\end{aligned}
$$


The energy-momentum tensor to which the five-dimensional Einstein's equations couple is given by (21). Since the brane action depends on $g_{\mu \nu}$ only through $\widetilde{g}_{\mu \nu}=e^{\beta \phi} g_{\mu \nu}$, we can write

$$
\frac{\partial \mathcal{L}_{4}}{\partial g^{\mu \nu}}=\frac{\partial \widetilde{g}^{\rho \sigma}}{\partial g^{\mu \nu}} \frac{\partial \mathcal{L}_{4}}{\partial \widetilde{g}^{\rho \sigma}}=-\frac{1}{2} e^{-\beta \phi} \sqrt{\widetilde{g}} \widetilde{T}_{\mu \nu} \delta(y) .
$$

Using $\sqrt{g}=n a^{3} b$ and $\sqrt{g}=e^{2 \beta \phi} n a^{3}$, from (21) we get

$$
\begin{aligned}
T_{a b}^{(4)} & =e^{-\beta \phi} \sqrt{\frac{\widetilde{g}}{g}} \widetilde{T}_{\mu \nu} \delta_{a}^{\mu} \delta_{b}^{\nu} \delta(y) \\
& =\frac{e^{\beta \phi}}{b} \widetilde{T}_{\mu \nu} \delta_{a}^{\mu} \delta_{b}^{\nu} \delta(y),
\end{aligned}
$$

or, more explicitly,

$$
\begin{aligned}
T_{00}^{(4)} & =\frac{n^{2}}{b} e^{2 \beta \phi} \tilde{\rho} \delta(y) \\
T_{i j}^{(4)} & =\frac{a^{2}}{b} e^{2 \beta \phi} \tilde{p} \delta(y) \delta_{i j},
\end{aligned}
$$

with other components vanishing.

Our primary interest is in the cosmological equation (39), where we are now in a position to evaluate the first derivatives of the metric coefficients $a$ and $n$ on the brane. These are derived using the general relation (37) applied to Einstein's equations $G_{a b}=M_{5}^{-3} T_{a b}$, with $G_{a b}$ given by (15-20) and the relevant components of $T_{a b}$ by (51-52). The jump conditions for the metric components then yield

$$
\begin{aligned}
& \frac{a_{0}^{\prime}}{a_{0}}=-\frac{1}{6 M_{5}^{3}} b_{0} e^{2 \beta \phi_{0}} \widetilde{\rho}, \\
& \frac{n_{0}^{\prime}}{n_{0}}=\frac{1}{6 M_{5}^{3}} b_{0} e^{2 \beta \phi_{0}}(2 \widetilde{\rho}+3 \widetilde{p}) .
\end{aligned}
$$

Finally we need the jump condition for $\phi$, derived from the equation of motion (29). In the case of conformal coupling the brane source for $\phi$ can be expressed in terms of $\tilde{\rho}$ and $\tilde{p}$ by using the fact that $\mathcal{L}_{4}$ depends on $\phi$ only through $\widetilde{g}_{\mu \nu}$ to write

$$
\begin{aligned}
\frac{\partial \mathcal{L}_{4}}{\partial \phi}=\frac{\partial \widetilde{g}^{\mu \nu}}{\partial \phi} \frac{\partial \mathcal{L}_{4}}{\partial \widetilde{g}^{\mu \nu}} & =\left(-\beta \widetilde{g}^{\mu \nu}\right)\left(-\frac{1}{2} \sqrt{\widetilde{g}} \widetilde{T}_{\mu \nu}\right) \delta(y) \\
& =-\frac{\beta}{2} \sqrt{\widetilde{g}}(\widetilde{\rho}-3 \widetilde{p}) \delta(y) .
\end{aligned}
$$

Putting it together gives

$$
\frac{\partial \widehat{\mathcal{L}}_{\phi}}{\partial \phi}=-\frac{\beta}{2} \sqrt{\frac{\widetilde{g}}{g}}(\widetilde{\rho}-3 \widetilde{p}) \delta(y)
$$


The $\phi$ equation is thus

$$
\square \phi=\frac{\beta}{4 \alpha} \frac{e^{2 \beta \phi}}{b}(\widetilde{\rho}-3 \widetilde{p}) \delta(y),
$$

where the D'Alembertian is given by (30). The jump equation for $\phi$ is therefore

$$
\phi_{0}^{\prime}=\frac{\beta}{8 \alpha} b_{0} e^{2 \beta \phi_{0}}(\widetilde{\rho}-3 \widetilde{p}) .
$$

Now we plug in the brane equations to (39), yielding

$$
\frac{\ddot{a}_{0}}{a_{0}}+\frac{\dot{a}_{0}^{2}}{a_{0}^{2}}=-\frac{1}{576 M_{5}^{6}} e^{4 \beta \phi_{0}}\left[\left(16+3 \frac{\beta^{2}}{\alpha} M_{5}^{3}\right) \widetilde{\rho}^{2}+\left(48-18 \frac{\beta^{2}}{\alpha} M_{5}^{3}\right) \tilde{\rho} \widetilde{p}+27 \frac{\beta^{2}}{\alpha} M_{5}^{3} \widetilde{p}^{2}\right]-\frac{\alpha}{3 M_{5}^{3}} \dot{\phi}_{0}^{2} .
$$

For a pure cosmological constant on the brane, we have $\widetilde{\rho}=-\widetilde{p}=\lambda$. Then (59) becomes

$$
\frac{\ddot{a}_{0}}{a_{0}}+\frac{\dot{a}_{0}^{2}}{a_{0}^{2}}=\frac{1}{36 M_{5}^{6}} e^{4 \beta \phi_{0}}\left(2-3 \frac{\beta^{2}}{\alpha} M_{5}^{3}\right) \lambda^{2}-\frac{\alpha}{3 M_{5}^{3}} \dot{\phi}_{0}^{2} .
$$

We see that this can vanish for any value of $\lambda$, if $\dot{\phi}_{0}=0$ and we choose

$$
\frac{\beta^{2}}{\alpha} M_{5}^{3}=\frac{2}{3} .
$$

This is the condition for self-tuning, as derived by [6, 7]. (Of course, making this choice is a kind of fine-tuning, as we discuss briefly in the Appendix.)

In this case, our cosmological equation (59) for more general matter sources becomes

$$
\frac{\ddot{a}_{0}}{a_{0}}+\frac{\dot{a}_{0}^{2}}{a_{0}^{2}}=-\frac{1}{32 M_{5}^{6}} e^{4 \beta \phi_{0}}(\widetilde{\rho}+\widetilde{p})^{2}-\frac{1}{2} \beta^{2} \dot{\phi}_{0}^{2} .
$$

If the brane tension is $\lambda$, we can decompose the energy density and pressure into tension plus dynamical energy-momentum (e.g. from matter and radiation on the brane): $\widetilde{\rho}=\lambda+\rho_{\mathrm{dyn}}$, $\widetilde{p}=-\lambda+p_{\mathrm{dyn}}$. From (62), however, the tension simply cancels out, and we are left with an identical equation relating for the dynamical density/pressure as we have for the total density/pressure. As this equation is quadratic rather than linear in the energy density, it will not yield anything close to conventional Friedmann behavior, and can't be taken seriously as a description of the real world. (We note that matter on the brane does not respond directly to the scale factor $a$, but to the conformally-transformed scale factor; however, for constant $\phi$ the functional dependence on the energy density will still be quadratic.) 


\subsection{Volume-element coupling}

In this section we consider a model in which $\phi$ enters only in an overall factor multiplying the Lagrange density, not in every appearance of the metric; in the notation of (11) we have

$$
\widehat{\mathcal{L}}_{4}=e^{2 \beta \phi} f\left(g_{\mu \nu}^{(4)}, \psi_{i}\right)
$$

This is equivalent to modifying the four-dimensional spacetime volume element:

$$
d^{4} x \sqrt{g^{(4)}} \rightarrow d^{4} x \sqrt{\left|e^{\beta \phi} g_{\mu \nu}^{(4)}\right|}=d^{4} x e^{2 \beta \phi} \sqrt{g^{(4)}}
$$

while otherwise coupling to the induced metric $g_{\mu \nu}^{(4)}$. Unlike the example of conformal coupling, this form of the action can be disrupted by quantum corrections; on the other hand, we shall see that the volume-coupling ansatz leads to a somewhat more acceptable cosmological model, and is worth exploring for that reason.

Since $\phi$ now couples non-universally to matter, it is most sensible to think of $g_{\mu \nu}$ as the metric to which matter responds, and the coupling to $e^{\beta \phi}$ as an interaction. The energymomentum tensor therefore takes the conventional form

$$
T_{\nu}^{\mu}=-2 \frac{1}{\sqrt{g^{(4)}}} g^{(4) \mu \lambda} \frac{\partial \mathcal{L}_{4}}{\partial g^{(4) \lambda \nu}}=\operatorname{diag}(-\rho, p, p, p) .
$$

This is related to $T_{a b}^{(4)}$, the brane contribution to the energy-momentum tensor appearing in the five-dimensional Einstein equations, by

$$
T_{a b}^{(4)}=-2 \frac{1}{\sqrt{g^{(5)}}} \frac{\partial \mathcal{L}_{4}}{\partial g^{(5) a b}}=\sqrt{\frac{g^{(4)}}{g^{(5)}}} T_{\mu \nu} \delta_{a}^{\mu} \delta_{b}^{\nu} .
$$

The specific components of $T_{a b}^{(4)}$ are thus:

$$
\begin{aligned}
T_{00}^{(4)} & =\frac{n^{2}}{b} \rho \delta(y) \\
T_{i j}^{(4)} & =\frac{a^{2}}{b} p \delta(y) \delta_{i j} .
\end{aligned}
$$

These differ from (51)-(52) of the previous section by the replacement $\left(e^{2 \beta \phi} \widetilde{\rho}, e^{2 \beta \phi} \widetilde{p}\right) \rightarrow(\rho, p)$. As a consequence, the jump conditions for the metric coefficients are

$$
\begin{aligned}
& \frac{a_{0}^{\prime}}{a_{0}}=-\frac{1}{6 M_{5}^{3}} b_{0} \rho, \\
& \frac{n_{0}^{\prime}}{n_{0}}=\frac{1}{6 M_{5}^{3}} b_{0}(2 \rho+3 p) .
\end{aligned}
$$


To express the jump condition for $\phi$ in terms of $\rho$ and $p$, we need to calculate $\partial \widehat{\mathcal{L}}_{4} / \partial \phi$. We can do this by considering our four-dimensional action to be that of a perfect fluid, corresponding to

$$
\widehat{\mathcal{L}}_{4}=e^{2 \beta \phi} f\left(g_{\mu \nu}^{(4)}, \psi_{i}\right)=p(\epsilon, s)-\frac{n}{2 \epsilon}\left(g_{\mu \nu}^{(4)} \Omega^{\mu} \Omega^{\nu}+\epsilon^{2}\right)
$$

(see [11, 13] for a discussion). In (71), the dynamical degrees of freedom with respect to which we vary the action to obtain equations of motion include the entropy $s$, the enthalpy $\epsilon$, a Lagrange multiplier $n$, the three Clebsch potentials $\chi, \alpha, \beta$, and the thermasy $\theta$; these last four scalars define the four-vector $\Omega^{\mu}$ via

$$
\Omega_{\mu}=\partial_{\mu} \chi+\alpha \partial_{\mu} \beta+\theta \partial_{\mu} s
$$

Note that varying with respect to $n$ gives the constraint $\Omega_{\mu} \Omega^{\mu}+\epsilon^{2}=0$; hence, on-shell the Lagrange density is simply equal to the pressure $p$. Therefore, from (29) the equation of motion for $\phi$ is

$$
\begin{aligned}
\square \phi & =-\frac{1}{2 \alpha} \frac{1}{b} \frac{\partial \widehat{\mathcal{L}}_{4}}{\partial \phi} \delta(y) \\
& =-\frac{\beta}{\alpha b} p \delta(y) .
\end{aligned}
$$

This corresponds to a jump condition describing the behavior of $\phi$ in the vicinity of the brane,

$$
\phi_{0}^{\prime}=-\frac{\beta}{2 \alpha} b_{0} p .
$$

Now we plug in these jump conditions to (39), yielding

$$
\frac{\ddot{a}_{0}}{a_{0}}+\frac{\dot{a}_{0}^{2}}{a_{0}^{2}}=-\frac{1}{36 M_{5}^{6}}\left(\rho^{2}+3 \rho p+3 \frac{\beta^{2}}{\alpha} M_{5}^{3} p^{2}\right)-\frac{\alpha}{3 M_{5}^{3}} \dot{\phi}_{0}^{2} .
$$

We see that the self-tuning condition is satisfied again only for

$$
\frac{\beta^{2}}{\alpha} M_{5}^{3}=\frac{2}{3},
$$

in which case our cosmological equation (76) for more general matter sources becomes

$$
\frac{\ddot{a}_{0}}{a_{0}}+\frac{\dot{a}_{0}^{2}}{a_{0}^{2}}=-\frac{1}{36 M_{5}^{6}}(\rho+p)(\rho+2 p)-\frac{1}{2} \beta^{2} \dot{\phi}_{0}^{2} .
$$


The contribution of the brane tension $\lambda$ to the Lagrangian enters in the combination $e^{2 \beta \phi_{0}} \lambda$. We therefore decompose the energy density and pressure into tension plus dynamical sources in the following manner:

$$
\begin{aligned}
& \rho=e^{2 \beta \phi_{0}} \lambda+\rho_{\mathrm{dyn}}, \\
& p=-e^{2 \beta \phi_{0}} \lambda+p_{\mathrm{dyn}} .
\end{aligned}
$$

Then, unlike the cosmological equation (62) obtained for conformal coupling, in the case of volume-element coupling we obtain terms on the right-hand side which are linear in the dynamical energy density and pressure:

$$
\frac{\ddot{a}_{0}}{a_{0}}+\frac{\dot{a}_{0}^{2}}{a_{0}^{2}}=\frac{1}{36 M_{5}^{6}}\left[e^{2 \beta \phi_{0}} \lambda\left(\rho_{\text {dyn }}+p_{\text {dyn }}\right)-\left(\rho_{\text {dyn }}^{2}+3 \rho_{\text {dyn }} p_{\text {dyn }}+2 p_{\text {dyn }}^{2}\right)\right]-\frac{1}{2} \beta^{2} \dot{\phi}_{0}^{2} .
$$

An equivalent equation was derived by Mennim and Battye [11].

An equation of this type stands a chance of describing the real world. The terms quadratic in energy/momentum can presumably be neglected at late times; if we assume that the $\dot{\phi}_{0}^{2}$ term is negligible we are left with a relation which has some resemblance to the hoped-for equation (7). With this in mind, we turn now to comparison with observation.

\section{Discussion}

Let us consider whether, under favorable circumstances, (81) could be consistent with what we know about the universe.

A time-dependent bulk scalar $\phi$ would generally lead to observable time-dependence in the four-dimensional Newton's constant, and therefore must be very small. Although the models under consideration do not include any mechanism for stabilizing $\phi$, we will proceed optimistically and imagine that the bulk scalar may be approximated as independent of time,

$$
\phi(y, t)=\bar{\phi}(y) .
$$

We imagine further that the $y$-dependence of the metric coefficients may be factored out, and the coefficient $b^{2}$ of $d y^{2}$ set to unity by an appropriate rescaling (which may always be done if $b^{2}$ is independent of $t$ ):

$$
d s^{2}=\omega(y)\left[-d t^{2}+a_{0}^{2}(t) d \vec{x}^{2}\right]+d y^{2} .
$$

Referring back to the jump conditions (70) and (75), such ansätze do not seem unreasonable; in (70) and (75) the symbols $\rho$ and $p$ refer to the entire energy and pressure, which will be 
dominated by the brane tension term, which in turn remains constant. Thus, it is reasonable to approximate the bulk solutions for $\bar{\phi}$ and $\omega$ by on their flat-space values as derived in [6, 7],

$$
\bar{\phi}(y)=\phi_{0}-\frac{1}{2 \beta} \ln [\omega(y)]
$$

and

$$
\omega(y)=\sqrt{1-y / y_{c}},
$$

where $y_{c}$ represents the location of the boundary opposite our brane, corresponding to a singularity,

$$
y_{c}=\frac{3 M_{5}^{3}}{2 \lambda} e^{-2 \beta \phi_{0}} .
$$

In order to compare the modified Friedmann relation (81) to observations, it is necessary to express the four-dimensional Planck mass $M_{4}=1 / \sqrt{8 \pi G_{4}}$ in terms of five-dimensional quantities. $M_{4}$ is defined by an integral over the extra dimension,

$$
M_{4}^{2}=M_{5}^{3} \int_{0}^{y_{c}} d y \omega(y)=\frac{M_{5}^{6}}{\lambda} e^{-2 \beta \phi_{0}} .
$$

(In fact, we are again ignoring a subtlety: due to the presence of the scalar field, general relativity is not exactly recovered in four dimensions, and the definition of $G$ has to be specified more carefully. It is possible that solar-system tests of gravity would rule out these simple models more definitively than the cosmological scenario considered here.)

For simplicity we drop the subscript "dyn" from $\rho$ and $p$ for the remainder of this section. In terms of the Hubble parameter $H=\dot{a} / a$, we have $\ddot{a} / a+(\dot{a} / a)^{2}=\dot{H}+2 H^{2}$. Then setting $\dot{\phi}_{0}=0$ and ignoring quadratic terms in $\rho, p$, our cosmological equation (81) becomes

$$
\dot{H}+2 H^{2}=\frac{2 \pi G}{9}(\rho+p) .
$$

Unlike the ordinary Friedmann equation, which is a constraint relating the value of the Hubble parameter to the energy density, this is a differential equation for $H$, which will involve an additional integration constant in its solution. In fact we can integrate (88) explicitly to obtain

$$
H^{2}=\frac{4 \pi G}{9} a^{-4} \int a^{3}(\rho+p) d a .
$$

Consider a universe dominated by a combination of matter $\left(\rho_{\mathrm{M}} \propto a^{-3}, p_{\mathrm{M}}=0\right)$ and radiation $\left(\rho_{\mathrm{R}} \propto a^{-4}, p_{\mathrm{R}}=\rho_{\mathrm{R}} / 3\right)$. The Hubble parameter then obeys

$$
H^{2}=\frac{4 \pi G}{9} \rho_{\mathrm{M}}+\frac{16 \pi G}{27} \rho_{\mathrm{R}} \ln \left(\frac{a}{a_{*}}\right),
$$


where $a_{*}$ is the integration constant alluded to above.

It is this relation (90) which is to be compared to the conventional Friedmann law $H^{2}=(8 \pi G / 3) \rho$. In a matter-dominated era, the functional dependence of $H$ on $a$ is conventional, leading to the familiar $a \propto t^{2 / 3}$ behavior. The coefficient of $\rho$, however, is different, suggesting a possible empirical test of the model: in this theory, the Hubble parameter during matter domination will be smaller by a factor of $1 / \sqrt{6} \approx 0.41$ than the conventional expectation (at fixed $\rho$ ). Unfortunately, we do not have very precise empirical information about the expansion rate during the matter-dominated era. Given that our current universe is apparently dominated by a smooth component causing it to accelerate, we cannot directly constrain the coefficient of $\rho_{\mathrm{M}}$ by contemporary observations. Since recombination occurred during the matter-dominated era, it would be possible in principle to constrain this coefficient via observations of the CMB anisotropy power spectrum; it is first necessary, however, to reliably calculate what that power spectrum should be, taking into account possible long-range deviations from general relativity due to the extra dimension. Since we have not undertaken this task, we are unable to say whether $H^{2}=(4 \pi G / 9) \rho_{\mathrm{M}}$ is consistent with the real universe.

In the radiation-dominated era, quantitative constraints on the behavior of the Hubble parameter may be derived from Big-Bang Nucleosynthesis (BBN) [12]. Expanding the logarithm in $(90)$, we have

$$
H_{\mathrm{R}}^{2}=\frac{16 \pi G}{27} \rho_{\mathrm{R}} \ln a+C a^{-4}
$$

where $C$ is an integration constant. This constant is not merely a nuisance that can be set to zero, but rather reflects the normalization of $a$; if we set $a=1$ today, the first term is always negative in the past, which is clearly unworkable. On the other hand, we can choose $C$ such that the second term is dominant — and with the correct magnitude to be compatible with observation - during BBN. This seems like an unlikely bit of fine-tuning, although it cannot be rigorously excluded. Therefore we see no way to definitively state that the self-tuning cosmologies we have considered are incompatible with observation, although it would require a mysterious coincidence to predict the correct light-element abundances. Since these models were invented to solve the fine-tuning problem associated with the cosmological constant, this must be considered as a strike against them.

Nevertheless, it is important to keep in mind that we have only dealt with specific toy models of self-tuning, which perhaps it is too optimistic to expect would lead to completely realistic cosmologies. The general idea that the cosmological constant problem may be solved not by making the vacuum energy small, but by making the metric insensitive to its value, is 
an interesting one, and it seems worth the effort to attempt to construct self-tuning models with more acceptable cosmological behavior.

\section{Acknowledgments}

We would like to thank Pierre Binetruy, Gregory Gabadadze, Steve Giddings, Monica Guica, Shamit Kachru, Nemanja Kaloper, Finn Larsen, Joe Lykken, Ricardo Rattazzi, Eva Silverstein, and David Wands for useful conversations. This work was supported in part by the U.S. Dept. of Energy, the Alfred P. Sloan Foundation, and the David and Lucile Packard Foundation.

\section{Appendix: Two observations}

In this Appendix we very briefly mention two issues of somewhat related interest to this work: first, the possibility of inflationary behavior in self-tuning cosmologies, and second, the ability to tune away other equations of state.

An obvious question which arises in any model which would make the metric insensitive to vacuum energy is, how are we to explain the apparent nonzero value of the cosmological constant today [3, 4], or implement an inflationary scenario in the early universe? Of course if a realistic self-tuning theory is found, it is conceivable that the currently observed vacuum energy is simply a reflection of an imperfectly tuned universe, once all aspects of the theory are taken into account. On the other hand, it is interesting to note that there is no difficulty in obtaining "accelerating" solutions in the presence of slowly-rolling scalar fields. Consider a minimally coupled four-dimensional scalar $\Phi$, with potential $V(\Phi)$. The energy density and pressure are given by

$$
\rho_{\Phi}=\frac{1}{2} \dot{\Phi}^{2}+V(\Phi), \quad p_{\Phi}=\frac{1}{2} \dot{\Phi}^{2}-V(\Phi)
$$

or

$$
\rho_{\Phi}+p_{\Phi}=\dot{\Phi}^{2}
$$

For a slowly-rolling scalar with $\dot{\Phi} \approx$ const, 89 then implies $H \approx$ const, just as in conventional theory with a nonzero vacuum energy. Thus there is no obstacle in principle to obtaining accelerated expansion either today or in the early universe. Of course the usual tuning problems associated with getting the correct nonzero value of the apparent vacuum 
energy are as severe in such a hypothetical model as they are in conventional quintessence and inflation scenarios.

Another interesting issue is the possibility of choosing parameters which work to tune away the effects of a more general energy component with equation of state $p=w \rho$, where $w$ is not necessarily -1 . We know of no compelling reason why this should happen, but the exercise illustrates the extent to which there really is some tuning going on in our choice of parameters.

Vacuum energy was tuned away by choosing our parameters $\alpha$ and $\beta$ to be related by (77) (taking the case of volume-element coupling for definiteness). Let us imagine that we instead take

$$
\frac{\beta^{2}}{\alpha} M_{5}^{3}=\frac{2}{3} x,
$$

where $x$ is a parameter to be chosen. The cosmological equation (76) becomes (setting $\dot{\phi}_{0}=0$ for simplicity),

$$
\frac{\ddot{a}_{0}}{a_{0}}+\frac{\dot{a}_{0}^{2}}{a_{0}^{2}}=-\frac{1}{32 M_{5}^{6}}\left(\rho^{2}+3 \rho p+2 x p^{2}\right) .
$$

For a universe dominated by a component with $p=w \rho$, the right hand side will automatically vanish if we choose

$$
x=-\frac{1+3 w}{2 w^{2}} .
$$

The choice $x=1$ tunes away vacuum energy $(w=-1)$ as well as an exotic component with $p=-\rho / 2$. With the exception of matter $(w=0)$, the expansion can be made insensitive to any specific equation of state by an appropriate choice of $x$. Once again, we have no reason to suggest that such a possibility will occur (nor see any need for it); however, this phenomenon serves as an illustration that the specific choice (77) represents a kind of fine-tuning in its own right.

\section{References}

[1] S. Weinberg, "The Cosmological Constant Problem," Rev. Mod. Phys. 61, 1 (1989).

[2] E. Witten, "The cosmological constant from the viewpoint of string theory," hep$\mathrm{ph} / 0002297$.

[3] S. M. Carroll, "The cosmological constant," Living Reviews in Relativity, 2001-1 (2001) astro-ph/0004075. 
[4] A. G. Riess et al. [Supernova Search Team Collaboration], Astron. J. 116, 1009 (1998) astro-ph/9805201; S. Perlmutter et al. [Supernova Cosmology Project Collaboration], Astrophys. J. 517, 565 (1999) astro-ph/9812133.

[5] N. Arkani-Hamed, S. Dimopoulos and G. Dvali, Phys. Lett. B 429, 263 (1998) hepph/9803315; I. Antoniadis, N. Arkani-Hamed, S. Dimopoulos and G. Dvali, "New dimensions at a millimeter to a Fermi and superstrings at a TeV," Phys. Lett. B 436, 257 (1998) hep-ph/9804398; K. R. Dienes, E. Dudas and T. Gherghetta, Phys. Lett. B 436, 55 (1998) hep-ph/9803466]; L. Randall and R. Sundrum, "An alternative to compactification," Phys. Rev. Lett. 83, 4690 (1999) hep-th/9906064.

[6] N. Arkani-Hamed, S. Dimopoulos, N. Kaloper and R. Sundrum, "A small cosmological constant from a large extra dimension," Phys. Lett. B480, 193 (2000) hepth/0001197].

[7] S. Kachru, M. Schulz and E. Silverstein, "Self-tuning flat domain walls in 5d gravity and string theory," Phys. Rev. D62, 045021 (2000) hep-th/0001206]; S. Kachru, M. Schulz and E. Silverstein, "Bounds on curved domain walls in 5d gravity," hep-th/0002121.

[8] P. Binetruy, C. Deffayet and D. Langlois, "Non-conventional cosmology from a braneuniverse," Nucl. Phys. B565, 269 (2000) hep-th/9905012.

[9] H. A. Chamblin and H. S. Reall, "Dynamic dilatonic domain walls," Nucl. Phys. B 562, 133 (1999) hep-th/9903225; C. Csaki, M. Graesser, C. Kolda and J. Terning, "Cosmology of one extra dimension with localized gravity," Phys. Lett. B 462, 34 (1999) hep-ph/9906513; J. M. Cline, C. Grojean and G. Servant, "Cosmological expansion in the presence of extra dimensions," Phys. Rev. Lett. 83, 4245 (1999) [hep-ph/9906523]; P. Kanti, I. I. Kogan, K. A. Olive and M. Pospelov, "Cosmological 3-brane solutions," Phys. Lett. B 468, 31 (1999) hep-ph/9909481; L. Mersini, "Decaying cosmological constant of the inflating branes in the Randall-Sundrum-Oda model," hep-ph/9909494; P. Binetruy, C. Deffayet, U. Ellwanger and D. Langlois, "Brane cosmological evolution in a bulk with cosmological constant," Phys. Lett. B 477, 285 (2000) hep-th/9910219; P. Kraus, "Dynamics of anti-de Sitter domain walls," JHEP9912, 011 (1999) thepth/9910149]; T. Shiromizu, K. Maeda and M. Sasaki, "The Einstein equations on the 3-brane world," Phys. Rev. D 62, 024012 (2000) gr-qc/9910076; E. E. Flanagan, S. H. Tye and I. Wasserman, "Cosmological expansion in the Randall-Sundrum 
brane world scenario," Phys. Rev. D 62, 044039 (2000) hep-ph/9910498; P. Kanti, I. I. Kogan, K. A. Olive and M. Pospelov, "Single-brane cosmological solutions with a stable compact extra dimension," Phys. Rev. D 61, 106004 (2000) [hep-ph/9912266]; L. Mersini, "Radion potential and brane dynamics," hep-ph/0001017.

[10] S. Forste, Z. Lalak, S. Lavignac and H. P. Nilles "A comment on self-tuning and vanishing cosmological constant in the brane world" Phys. Lett. B481, 360 (2000) hep-th/0002164]; C. Csaki, J. Erlich, C. Grojean and T. Hollowood, "General properties of the self-tuning domain wall approach to the cosmological constant problem," Nucl. Phys. B584, 359 (2000) hep-th/0004133; G. T. Horowitz, I. Low and A. Zee, "Self-tuning in an outgoing brane wave model," hep-th/0004206; B. Grinstein, D. R. Nolte and W. Skiba, "Adding matter to Poincare invariant branes," hepth/0005001; C. Zhu, "A self-tuning exact solution and the non-existence of horizons in in 5d gravity-scalar systems," JHEP0006, 034 (2000) hhep-th/0005230; V. Barger, T. Han, T. Li, J. D. Lykken and D. Marfatia, "Cosmology and hierarchy in stabilized warped brane models," Phys. Lett. B488, 97 (2000) hep-ph/0006275; P. Binetruy, J. M. Cline and C. Grojean, "Dynamical instability of brane solutions with a self-tuning cosmological constant," hep-th/0007029; K. Maeda and D. Wands, "Dilaton-gravity on the brane," hep-th/0008188; L. E. Mendes and A. Mazumdar, "Brans-Dicke brane cosmology," gr-qc/0009017; Z. Kakushadze, "Self-tuning' and conformality," Mod. Phys. Lett. A15, 1879 (2000) hep-th/0009199; C. Kennedy and E. M. Prodanov "Standard cosmology on a self-tuning domain wall," hep-th/0010202; A. Kehagias and K. Tamvakis, "A self-tuning solution of the cosmological constant problem," hep-th/0011006; J. E. Kim, B. Kyae and H. M. Lee "A model for self-tuning the cosmological constant," hep-th/0011118; T. Padmanabhan and S. Shankaranarayanan, "Vanishing of cosmological constant in nonfactorizable geometry," Phys. Rev. D 63, 105021 (2001) hep-th/0011159]; J. E. Kim, B. Kyae and H. M. Lee "Self-Tuning Solution of the cosmological constant problem with antisymmetric tensor field," hep-th/0101027; P. Brax and A. C. Davis, "Cosmological evolution on self-tuned branes and the cosmological constant," hep-th/0104023.

[11] A. Mennim and R. A. Battye, "Cosmological expansion on a dilatonic brane-world," hep-th/0008192.

[12] K. A. Olive, G. Steigman and T. P. Walker, "Primordial nucleosynthesis: Theory and observations," Phys. Rept. 333-334, 389 (2000) astro-ph/9905320; S. Burles, 
K. M. Nollett, J. N. Truran and M. S. Turner, "Sharpening the predictions of big-bang nucleosynthesis," Phys. Rev. Lett. 82, 4176 (1999) astro-ph/9901157]; D. Tytler, J.M. O'Meara, N. Suzuki and D. Lubin, astro-ph/0001318.

[13] D. S. Salopek and J. M. Stewart, Class. Quant. Grav. 9, 1943 (1992). 